\title{
AL-FEC for streaming services in LTE E-MBMS
}

\author{
Jorge Calabuig, Jose F Monserrat", David Gozálvez and David Gómez-Barquero
}

\begin{abstract}
3rd Generation Partnership Project specified Application Layer - Forward Error Correction (AL-FEC) to be used for Enhanced Multimedia Broadcast Multicast Services (E-MBMS) in Long Term Evolution (LTE) networks. Specifically, Raptor coding is applied to both streaming and file delivery services. This article focuses on streaming services and investigates the optimum configuration of the AL-FEC mechanism depending on the signal-to-interference plus noise power ratio conditions. These configurations are compared with a scenario without an application layer protection to obtain the potential gain that can be achieved by means of AL-FEC. This article also studies the multiplexing of services within the AL-FEC time interleaving. These analyses were performed using a proprietary system level simulator and assuming both pedestrian and vehicular users. Different quality criterions were used to ensure the completeness of the study. Results show the significant benefit of using AL-FEC in E-MBMS in terms of coverage and service quality.
\end{abstract}

Keywords: LTE, E-MBMS, Raptor codes, AL-FEC, Broadcast, Streaming

\section{Introduction}

Universal mobile telecommunications systems (UMTS) integrated in March 2005-Release 6-Multimedia Broadcast Multicast Services (MBMS) as a technical feature to provide an efficient delivery mode of mass multimedia services such as mobile TV [1]. Since these services are designed to be simultaneously broadcasted to a large number of users, MBMS introduced new point-to -multipoint (p-t-m) radio bearers and multicast support in the core network. The main benefit of $\mathrm{p}-\mathrm{t}-\mathrm{m}$ bearers is the efficient usage of physical resources. Per contra, p-t-m bearers cannot adapt modulation and coding schemes for each individual user. This can result in high loss rates for users with poor channel conditions [2].

To overcome these problems and increase the robustness of the $\mathrm{p}-\mathrm{t}-\mathrm{m}$ transmissions, MBMS introduced an additional forward error correction (FEC) mechanism at the application layer (AL-FEC) based on Raptor codes for both streaming and file delivery services [3]. Raptor codes are a computationally efficient implementation of fountain codes that achieve close to ideal performance. This permits a software implementation without the need of dedicated hardware even in handheld devices [4]. By working at the application layer, it is possible to

\footnotetext{
* Correspondence: jomondel@iteam.upv.es

iTEAM Research Institute, Universitat Politècnica de València, Camino de Vera S/N, 46022, Valencia, Spain
}

recover packet losses of all underlying layers and protocols, providing end-to-end error correction without any required change in legacy standards.

In Release 7, the 3rd Generation Partnership Project (3GPP) standardized the use of MBMS over a Single Frequency Network (MBSFN), which boosts performance [5]. In a SFN, multiple base stations are synchronized and transmit the same information to the receivers. This improves signal quality reception and hence efficiency.

Later on, the 3GPP initiated the Long Term Evolution (LTE) standardization activity to enhance UMTS, thanks to the usage of Orthogonal Frequency Division Multiple Access (OFDMA) modulation [6]. The first version of LTE-Release 8-was completed in December 2008. LTE comprised from the beginning the MBMS feature, renamed as Enhanced MBMS (E-MBMS), whose definition in Release 8 only included physical channels [7]. It was not until Release 9-issued at the end of 2009when higher layer aspects were specified [8]. E-MBMS includes again SFN and AL-FEC mechanisms and adds new features inherited from other successful broadcast networks, such as the use of extended cyclic prefix (CP).

To this date, MBMS has been incorporated to a very limited number of commercial networks. However, with the better system performance and the higher flexibility provided by LTE, E-MBMS has regained interest $[9,10]$. 
Concerning the application of AL-FEC protection for broadcast and multicast services over wireless mobile networks, this topic has extensively been studied in 3rd Generation (3G) including file delivery [11-13] and streaming services $[13,14]$. These works analyzed the system trade-offs between AL-FEC and PHY-FEC for MBMS and concluded that only a well-designed system that combines both coders can maximize efficiency and user perception. The results also pointed out that the relationship between encoding rates defined in MBMS is not the optimal. It would be beneficial to reduce the protection at the physical layer and increase Raptor code protection. However, the protection period must be taken into account in case of streaming services to guarantee small zapping times, which is defined as the time elapsed since the viewer presses the channel change button until the new channel is displayed.

Regarding LTE and the application of AL-FEC in EMBMS, Alexiou et al. [15] presented a cost analysis that compares the performance of a new error recovery scheme based on Raptor codes with other existing approaches under different MBSFN deployments, user populations, and error rates. This study only focused on the file delivery service. Concerning streaming services, Munaretto et al. [16] analyzed a cross-layer framework aimed at optimizing the number of streamed video layers, the modulation and coding scheme (MCS), and the application layer FEC used for each layer. Bouras et al. [17] also studied the use of ALFEC over E-MBMS streaming services. Assuming a singlecell scenario, they investigated how the FEC overhead can be reduced under different packet loss conditions. Despite these two seminal works, the potential gain that can be achieved using AL-FEC mechanisms in E-MBMS has not been addressed in a holistic manner.

Note that all these works considered the 3GPP standardized Raptor coding [3]. However, there exists a new generation of fountain codes called RaptorQ [18]. This code minimizes the redundant FEC information outperforming Raptor code. Per contra, the improved coding performance comes at the expense of increased encoding and decoding complexity. Mladenov et al. [19] investigated the application of RaptorQ in MBMS services and compared Raptor and RaptorQ codes. They concluded that although the RaptorQ code has better coding properties, it is too complex for efficient real-time decoding in MBMS terminals.

This article evaluates the benefits of AL-FEC in streaming services over LTE MBSFN networks and provides guidelines for the efficient transmission of EMBMS services. Consequently, this study addresses simultaneously the radio resource management (RRM) problem [20] and the trade-off between PHY-FEC and AL-FEC. The main goal is to obtain the best configurations that allow the highest E-MBMS service data rate when a fixed amount of resources is allocated for E-
MBMS. On the other hand, this article also studies how the conditions of the scenario in terms of signal-to-interference plus noise power ratio (SINR) affect the selection of PHY-FEC and AL-FEC parameters. Finally, different alternatives of E-MBMS scheduling are evaluated to determine how different services must be multiplexed within the AL-FEC interleaving time. Extensive simulations have been carried out using a proprietary LTE-compliant system level simulator that has been extended to assess AL-FEC protection. It is worth noting that the simulation tool was calibrated against 3GPP and International Telecommunication Union Radiocommunication Sector (ITU-R) reports.

The remainder of this article is organized as follows: Section 2 describes the main features of E-MBMS included in the 3GPP LTE standard. Section 3 presents the FEC techniques used in E-MBMS. Section 4 describes the simulation environment and assumptions. Finally, simulation results are discussed in Section 5, whereas Section 6 draws the main conclusions of the article.

\section{E-MBMS technical features}

LTE E-MBMS includes some features previously used in 3G MBMS, such as MBSFN operation and AL-FEC, but E-MBMS also incorporates other new mechanisms. This section addresses these new features.

\subsection{Physical layer features}

In MBSFN operation, a cluster of time-synchronized cells transmits the same E-MBMS content. These cells form an MBSFN area and, in order to preserve orthogonality, signals from the MBSFN cells must reach the users within the CP. Otherwise, the signal produces inter-symbol interference. In order to allow larger cluster sizes, the LTE standard increases the CP from $4.6 \mu \mathrm{s}-$ normal $\mathrm{CP}$ - to $16.7 \mu \mathrm{s}$-extended $\mathrm{CP}$-for the MBSFN physical channel, the physical multicast channel (PMCH) [7]. Moreover, an optional double CP length of around $33 \mu$ s can be used in scenarios with large inter-site distances (ISDs). In order to avoid an increase in overhead due to the double-sized $\mathrm{CP}$, the number of subcarriers is also doubled by defining a subcarrier spacing of $7.5 \mathrm{kHz}$ instead of $15 \mathrm{kHz}$, only for MBMS-dedicated cells. However, this dedicated carrier deployment is still not supported in current releases of LTE and E-MBMS services must share resources with unicast users [8].

On the other hand, in E-MBMS the transmission time interval (TTI), which fixes the amount of information to be coded by the PHY-FEC mechanism, is $1 \mathrm{~ms}$. In this way, time interleaving at the E-MBMS physical layer is much lower than in the case of 3G MBMS, with TTIs from 10 up to $80 \mathrm{~ms}$. However, the OFDMA modulation used in LTE enables frequency diversity at the receiver, which is not present at the physical layer of 3G MBMS. 


\subsection{E-MBMS scheduling}

Whereas 3G MBMS services are multiplexed with unicast services using different channelization codes, LTE E-MBMS services are multiplexed in time inside MBSFN subframes. Therefore, some E-MBMS control information must be used in order to inform users about E-MBMS scheduling [21]. The logical channels that carry information of E-MBMS services are the multicast control channel $(\mathrm{MCCH})$ and the multicast traffic channel (MTCH). Traffic data of E-MBMS services are transmitted using an $\mathrm{MTCH}$, while $\mathrm{MCCH}$ carries control information associated to all MTCHs transmitted in a MBSFN area. Both MTCH and MCCH are mapped into the multicast transport channel $(\mathrm{MCH})$. Finally, the $\mathrm{MCH}$ transport channel is mapped into the $\mathrm{PMCH}$.

The eNodeB transmits System Information Blocks (SIBs) through the broadcast control channel (BCCH) [22]. There are two SIBs related to E-MBMS: SIB2 and SIB13. SIB2 informs the user about which subframes are reserved for MBSFN. On the other hand, SIB13 indicates how many MBSFN areas are configured in a cell and which are the subframes and the MCS used by the $\mathrm{MCCH}$ of each MBSFN area. MCCHs are periodically transmitted over one of the MBSFN-capable subframes, being this period broadcasted over the SIB13. It is important to note that among the 10 subframes included

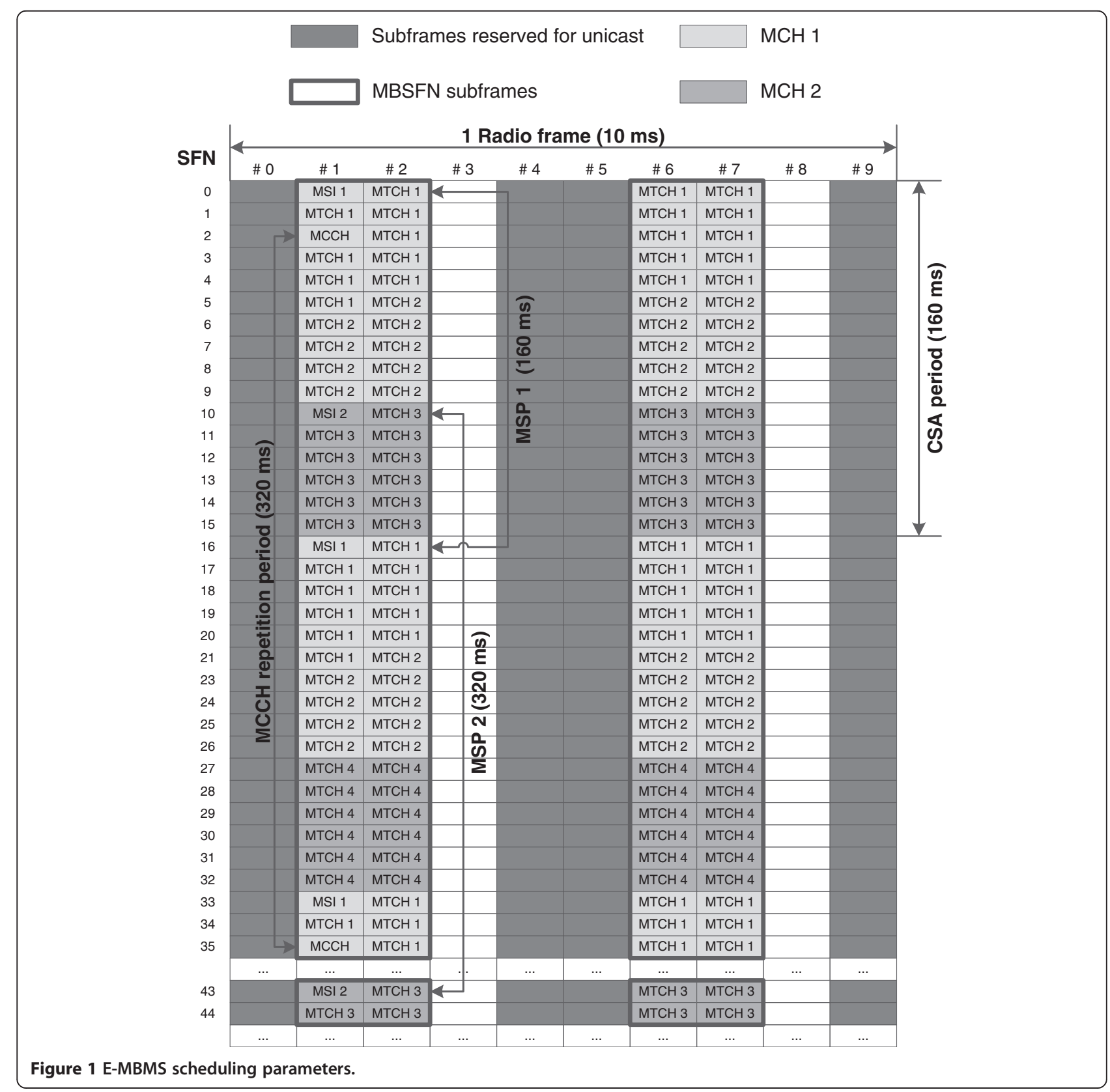


in a radio frame, the maximum number of MBSFN subframes is six, since subframes \#0, \#4, \#5, and \#9 are reserved for unicast transmission.

The $\mathrm{MCCH}$ informs about the scheduling of the PMCHs within the MBSFN subframes related to this MBSFN area through the Common Subframe Allocation (CSA) and the PMCH-InfoList. The former indicates which subframes are reserved for all the $\mathrm{MCHs}$ of an MBSFN area, while the latter indicates how the subframes are shared among those MCHs. Besides, $\mathrm{PMCH}-$ InfoList reports the MCS of the E-MBMS services related to each $\mathrm{MCH}$. Finally, each $\mathrm{MCH}$ can multiplex several E-MBMS services. In order to identify the specific E-MBMS service, the PMCH-InfoList defines all the EMBMS ongoing sessions (identified by MTCH). Moreover, the PMCH-InfoList indicates a period of time known as $\mathrm{MCH}$ Scheduling Period (MSP) where the associated MTCHs are multiplexed. This MTCH multiplexing is indicated in the first subframe of each MSP by means of a medium access control (MAC) control element called $\mathrm{MCH}$ Scheduling Information (MSI).

In order to clarify E-MBMS scheduling, Figure 1 shows an example of E-MBMS configuration. Four subframes are assigned to E-MBMS: \#1, \#2, \#6, and \#7. The repetition period of the $\mathrm{MCCH}$ is $320 \mathrm{~ms}$. The CSA period where two different $\mathrm{MCH}$ are scheduled is 160 ms. Each $\mathrm{MCH}$ has two E-MBMS services: MTCH 1 and MTCH 2 correspond to MCH 1 while MTCH 3 and MTCH 4 correspond to MCH 2. The MSPs are 160 and $320 \mathrm{~ms}$ for $\mathrm{MCH} 1$ and $\mathrm{MCH} 2$, respectively. Note that SFN in Figure 1 means system frame number.

\section{FEC techniques in E-MBMS}

As any communication system, LTE also employs FEC techniques. FEC mechanisms rely on the transmission of repair information to protect from packet losses in underlying levels without a need for feedback, in such a way that the receiver can detect and possibly correct errors occurred during transmission. In particular, LTE uses Turbo codes as the FEC mechanism that works at the physical layer and, for broadcast and multicast transmissions, LTE can use Raptor codes at the application layer [3].

LTE standard defines Turbo codes as the physical layer FEC to protect data against errors in the transmission over unreliable or noisy communication channels, such as mobile wireless channel. One of the main benefits of the use of turbo codes is that they can exploit channel state information using soft decision decoding, thus achieving a high-performance. However, in practice, due to on-chip memory and decoding complexity constraints, the maximum time interleaving depth is rather small. In LTE E-MBMS transmissions, this maximum time interleaving depth depends on the TTI or subframe length, which is only $1 \mathrm{~ms}$ [7]. As a consequence, PHYFEC is often combined with an AL-FEC code, thus achieving a better trade-off between overall system error protection and complexity of the implementation. By working at the application layer, it is possible to provide protection against longer losses with larger interleaving depths. However, the system FEC configuration must be optimized taking into account this cross-layer operation.

Figure 2 shows the protocol stack of E-MBMS including AL-FEC protection for the specific case of streaming services. AL-FEC coding is performed over Real-time Transport Protocol (RTP) packets. In video streaming applications, these RTP packets generally include H.264 Network Abstraction Layer (NAL) units and/or audio packets. In order to fit to the Maximum Transmission Unit (MTU) of the Internet Protocol (IP) layer, the NAL units might be fragmented.

The application of AL-FEC to streaming media in MBMS is described in [3]. As depicted in Figure 2, three parameters have to be defined with regards to Raptor encoding: the protection period $\left(T_{\mathrm{pp}}\right)$, the code rate $(k / n)$ and the source symbol size $(T)$ measured in bytes. The code rate $(\mathrm{CR})$ determines the amount of erroneous symbols than can be corrected at the application layer. Lower CRs increase not only the protection of AL-FEC, but also the amount of overhead that must be transmitted in the form of parity packets. On the other hand, $T_{\mathrm{pp}}$ determines the period of time over which the source blocks are transmitted. Longer protection periods imply higher time diversity and hence offer better robustness but at the cost of increasing the end-to-end delay and zapping time experimented by the user. This zapping time has a significant impact on the quality-of-service (QoS) perceived by the users. In order to reduce zapping time, several fast zapping techniques can be applied [3]. However, it must be noted that long protection periods greater than $10 \mathrm{~s}$ are not feasible in practice for streaming services, as they will involve long zapping times that

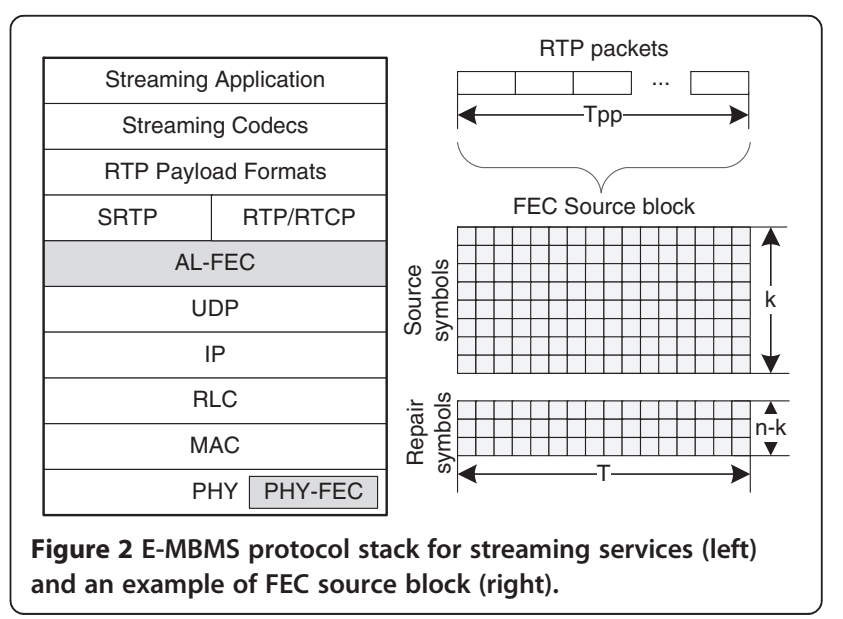




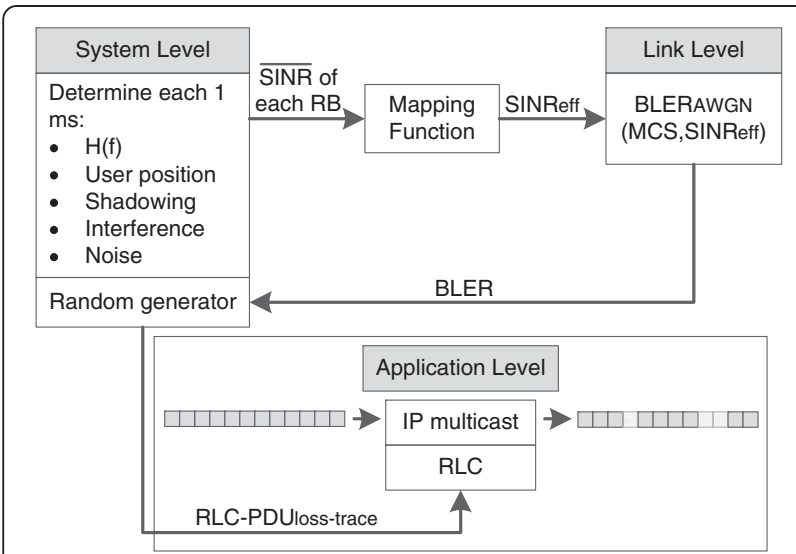

Figure 3 AL-FEC streaming simulator architecture.

would be difficult to tolerate by the users even with fast zapping techniques [23].

The Raptor coding process is based on a systematic Raptor encoder, which uses a source block of $k$ source symbols to generate the repair symbols. Then, first of all, a FEC source block is constructed from a set of RTP packets, exactly those generated in a $T_{\mathrm{pp}}$. The size of the FEC source block is $k$ times $T$, where $k$ is the number of source symbols (which also depends on the $T_{\mathrm{pp}}$ ). The selection of $T_{\mathrm{pp}}$ depends mainly on the desired delay and the memory available in the device. Therefore, all packets included in a single FEC source block are jointly protected. Afterwards, the Raptor encoder generates $n-$ $k$ repair symbols of size $T$ from the FEC source block according to the Raptor code rate.

After Raptor coding, two types of IP packets are obtained: FEC source packets and FEC repair packets. A source packet encapsulates original User Datagram Protocol packets while a repair packet encapsulates one or more repair symbols, which are generated in the FEC encoding process. Each source and repair packet contains additional information for the packets-to-block mapping. This way, a receiver can use Raptor decoding to recover a source block if enough encoding symbols are received for that source block.

\section{Simulation environment and assumptions}

Performance evaluation of AL-FEC for streaming services in E-MBMS was conducted by means of simulations. Specifically, simulations were divided into three levels of abstraction known as link, system, and application-level simulations. Following the study presented in [13], the first two levels are used to generate Radio Link ControlPacket Data Unit (RLC-PDU) loss traces, which are then used in the application-level simulations. Figure 3 shows the modular architecture used for the global simulations, where the three levels of abstraction can be identified.

\subsection{Link and system level simulations}

Link-level simulations are used to assess the performance of the physical layer and those MAC aspects directly related to the radio interface. At the link level, a continuous radio link is modeled, including simulationspecific features like modulation, channel coding, channel fading, channel estimation, demodulation, etc. In order to assess LTE E-MBMS physical layer, several linklevel simulations were carried out. These simulations were calibrated in the framework of the WINNER+ project, one of the International Mobile TelecommunicationsAdvanced (IMT-Advanced) evaluation groups of the ITU$\mathrm{R}$ [24].

On the other hand, system level simulations are used to evaluate the performance of a global network. At this level, system modeling encompasses a set of base stations and all their associated user terminals. The signal level received by each user, as well as other users' interferences, is modeled taking into account the propagation losses and channel fading effects. The SINR values can be translated to any link performance indicator, such as the block error rate (BLER), packet error rate (PER) or throughput values, employing link abstraction models that translate the results obtained at link level. In this article, a detailed modeling of multipath fading was performed.

Link abstraction models are developed using the linklevel simulations. The goal of these models is to allow the system level simulator to predict the performance of a link between a base station and a mobile terminal given a measure of the channel quality such as the SINR. In this article, the Mutual Information Effective SINR Mapping model was chosen [25]. Using this model, the multiple SINRs obtained at system level for a specific

Table 1 Main parameters of the scenario

\begin{tabular}{ll}
\hline Parameter & Value \\
\hline Number of sites & 19 sites (3 sectors per site). \\
Wrap-around layout & 19 cells \\
Number of MBSFN cells & $10 \mathrm{MHz}$ (50 RBs) \\
Bandwidth & $2 \mathrm{GHz}$ \\
Central frequency & $500,1500 \mathrm{~m}$ \\
ISD & $46 \mathrm{dBm}$ \\
Transmission power & $12^{\circ}$ \\
Antenna tilt & $-174 \mathrm{dBm} / \mathrm{Hz}$ \\
Noise spectral density & $9 \mathrm{~dB}$ \\
Mobile terminal noise & \\
figure & $20 \mathrm{~dB}$ \\
Penetration loss & According to IMT-A Urban \\
Large scale channel & Macro scenario \\
model parameters & Tapped delay line with EPA \\
Multipath channel model & $(3 \mathrm{~km} / \mathrm{h}$ ) and EVA (30 km/h) PDPs \\
\end{tabular}


Table 2 Main parameters of the application-level simulation

\begin{tabular}{ll}
\hline Parameter & Values \\
\hline AL-FEC CR & $1 / 5,1 / 4,1 / 3,3 / 8,2 / 5,9 / 20,1 / 2,11 / 20,3 / 5,2 / 3,7 / 10$, \\
& $3 / 4,5 / 6,7 / 8,23 / 25,1$ (no AL-FEC) \\
Protection period & $0.32,0.64,0.96,1.28,1.6,1.92,2.24,2.56,2.88,3.2$, \\
(s) & $3.52,3.84,4.16,4.48,4.8,5.12,5.44,5.76,6.08,6.4$ \\
MCS & QPSK: 120/1024, 193/1024, 308/1024, 449/1024, 602/1024 \\
MCS & 16-QAM: 378/1024, 490/1024, 616/1024 \\
\hline
\end{tabular}

link are translated to an effective SINR value in a first step and later converted to a BLER value in a second step. The latter step requires the availability of SINR to BLER mappings in AWGN conditions for each MCS. These mappings were obtained through link-level simulations. Finally, based on this BLER values, a random generator decides whether the included RLC/MAC block is received free of errors. The obtained results after system simulations are an RLC-PDU loss trace of $30 \mathrm{~min}$ for each user.

\subsection{Simulation scenario}

ITU guidelines for the IMT-Advanced candidate evaluation [26] were used as the main reference. The cell layout consisted of 57 cells, that is, 19 tri-sector sites. Within these 57 cells, only the 19 central cells formed the MBSFN area. A macro-cellular scenario was assumed since this scenario fits well with the expected deployment scenario of an E-MBMS network. Therefore, shadowing and path loss parameters correspond to those of the IMT-Advanced Urban Macro scenario in [26]. Antenna pattern took into account azimuth, elevation, and tilt [26]. In addition, the multipath channel was modeled using a tapped delay line model based on the Extended Pedestrian A (EPA) and Extended Vehicular A (EVA) Power Delay Profiles (PDPs) [27] for pedestrian users and vehicular users, respectively. The multipath channel model was only used for those cells belonging to the MBSFN area. Frequency-flat fading channels were assumed for the remaining cells. Table 1 presents the more important parameters of the scenario.

In MBSFNs, the interference consists of both external interferences and self-interference from the own network. Signals received within the OFDM symbol guard interval are considered as useful and contribute totally to the useful signal, whereas signals with a time delay greater than the guard interval cause self-interference. In practice, signals arriving with a delay slightly greater than the guard interval contribute partially to the useful signal and partially to the self-interference. In order to determine the ratio between the useful and interfering contribution, in this article the weighting function defined in [28] was used. On the other hand, external interferences come from the cells surrounding the MBSFN area. For the sake of simplicity, active users were only deployed within the MBSFN area, while surrounding cells were working at full capacity, that is, the total transmission power was distributed uniformly within the whole bandwidth.

In system level simulations, MBSFN area and EMBMS service reception area were differentiated in the same way as it is proposed in [29]. This was made in order to avoid a sharp drop in SINR due to users in the border of the MBSFN area. For an MBSFN area of 19 cells, E-MBMS service reception was studied within a radius equal to the ISD. Finally, pedestrian and vehicular users moved inside the E-MBMS service reception area and bounced at the edge of the circumference. In addition, a large amount of users-4,000 for an ISD of $500 \mathrm{~m}$ and 9,000 for an ISD of $1500 \mathrm{~m}$-were uniformly distributed within the E-MBMS area in order to ensure statistical significance of the results.

\subsection{Application-level simulations}

After system level simulations, the resulting RLC-PDU loss traces were applied to an IP multicast stream in the application-level simulator, where different Raptor

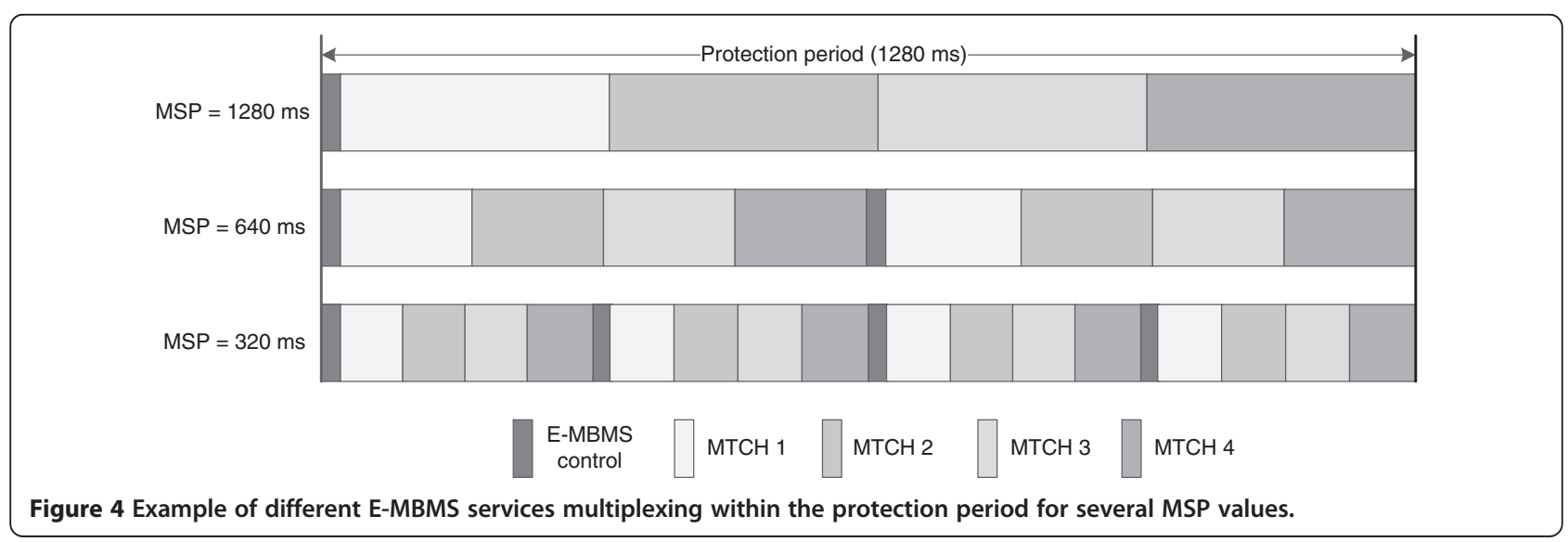



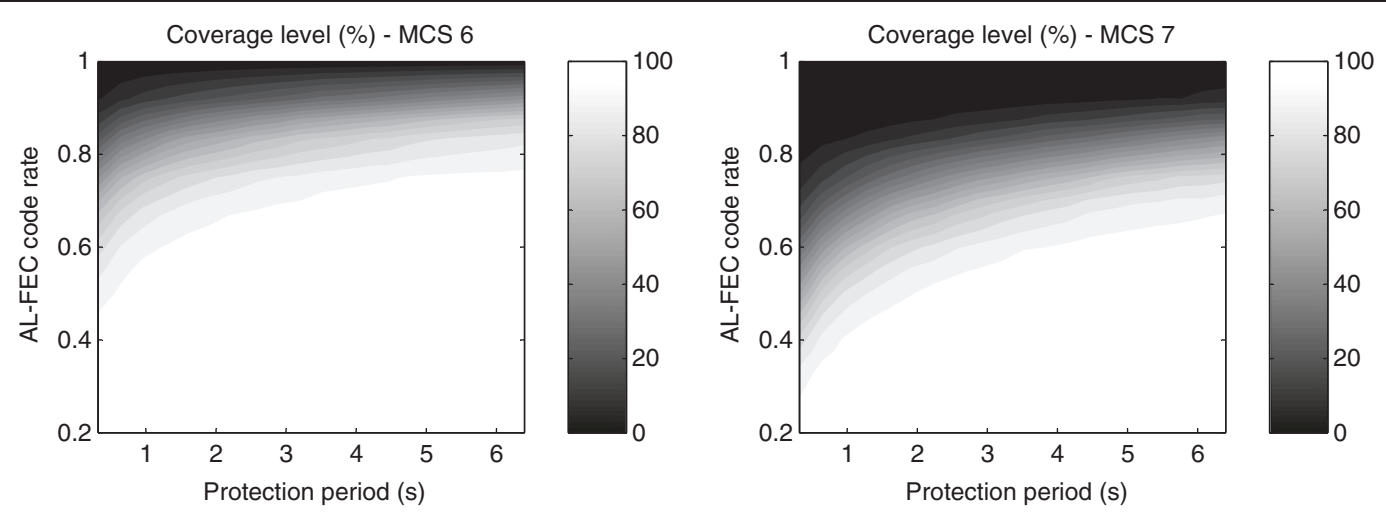

Figure 5 Coverage level versus AL-FEC CR and Tpp for MCS 6 (left) and MCS 7 (right).

coding parameters were studied. For the sake of simplicity, simulations assumed ideal Raptor coding, that is, if the total number of IP packets correctly received within a protection period-source and repair packets-is greater than or equal to the number of source packets, then original data are recovered. A fixed IP packet size of 1,024 bytes was used for both source packets and repair packets. Besides, given this IP packet size and the packet headers, RTP packet size is 992 bytes, which implies a source symbol size $T$ of 995 bytes.

In the LTE system, E-MBMS services consumed 40\% of resources, that is, four subframes per frame. MSP was set to $320 \mathrm{~ms}$. With this configuration, results were obtained for several combinations of protection period, application layer code rate, and MCS at the physical layer. Each combination of these parameters corresponds to an available service data rate.

Protection periods ranged from $320 \mathrm{~ms}$ to $6.4 \mathrm{~s}$ in multiples of the MSP. At the application layer, several code rates from $1 / 5$ up to the no protection-code rate 1 -were used. With respect to the physical layer, the data rates correspond to a set of MCS defined in the channel quality indicator table of LTE [30]. At the physical layer, if the transport block size is larger than the maximum code block size-6,144 bits-then it is fragmented in several turbo code blocks. In order to sum up, Table 2 presents all possible values for the main parameters used in the application-level simulations.

For the sake of clarity, Figure 4 shows an example of multiplexing of four streaming channels within an ALFEC protection period of $1.28 \mathrm{~s}$ taking into account different values of the MSP.

\section{Simulation results}

Several metrics can be specified to obtain the performance of each configuration. In this study, three metrics were used: the IP Packet Error Ratio (IP PER), the Erroneous Second Ratio (ESR), and the ESR5(20). The former represents the percentage of erroneously received IP packets. IP PER only account for the overall transmission errors experienced by the users and it does not represent the time distribution of the errors, which also affects the QoS of a streaming service perceived by the users. This can be taken into account with the other two metrics. ESR represents the percentage of seconds that contain errors and ESR5(20) represents the percentage of time intervals of $20 \mathrm{~s}$ with at most $1 \mathrm{~s}$ with errors (i.e., $5 \%$ errors).
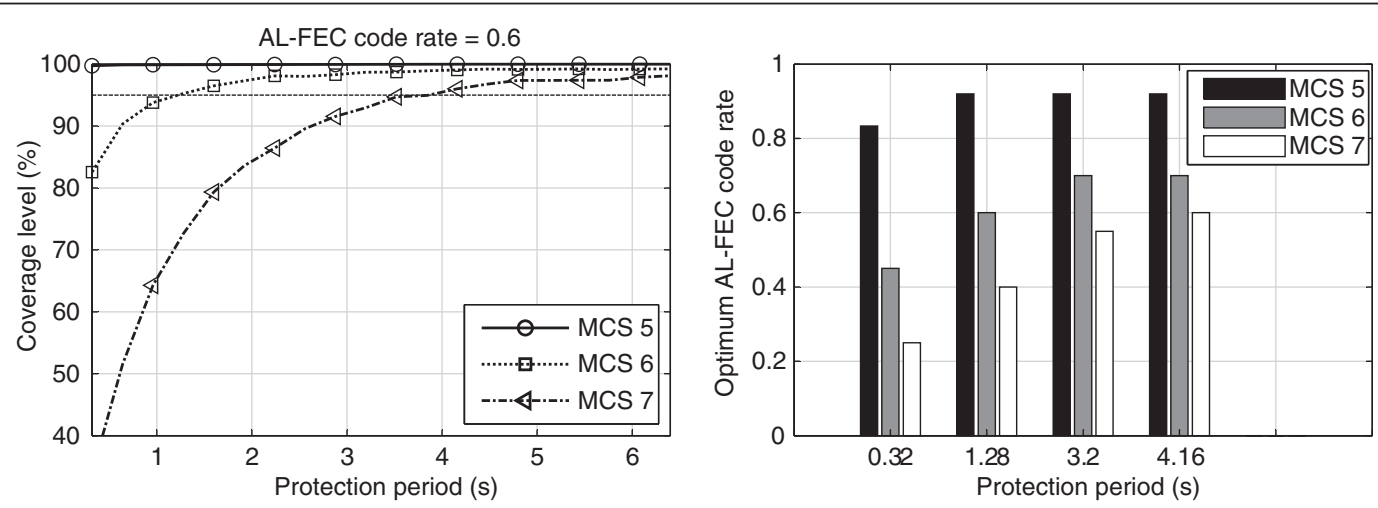

Figure 6 Coverage level versus $T_{\mathrm{pp}}$ for MCSs with fixed AL-FEC CR (left) and optimum AL-FEC CR versus $T_{\mathrm{pp}}$ for MCSs (right). 

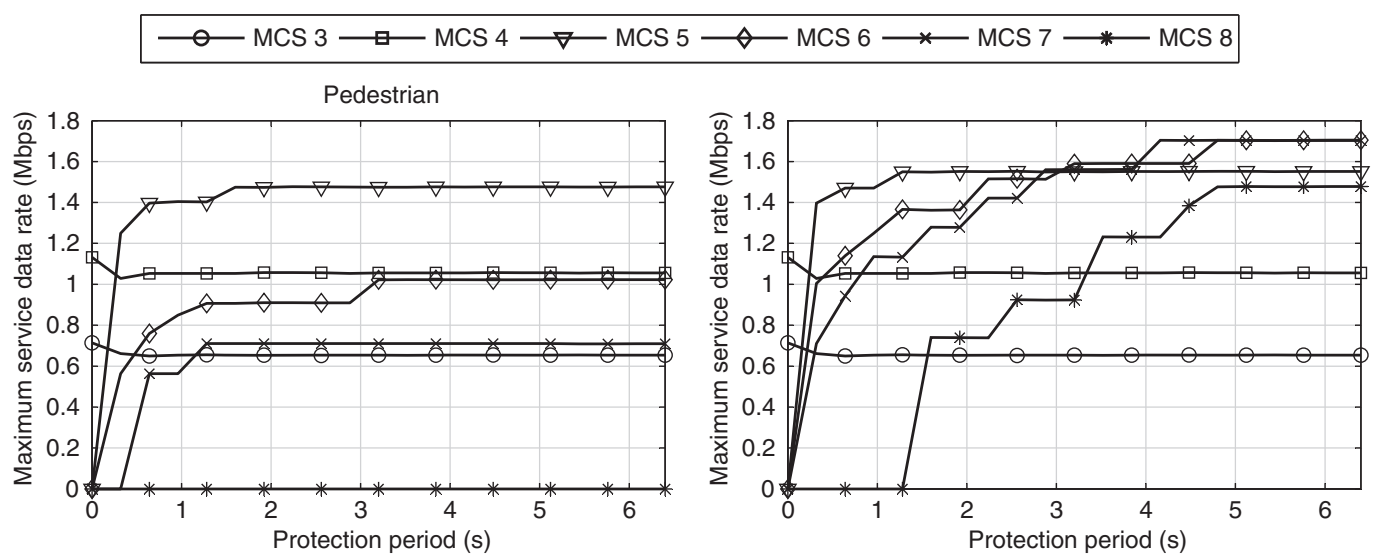

Figure 7 Maximum service data rate versus $T_{\mathrm{pp}}$ for MCSs with ESR metric for pedestrian (left) and vehicular (right) users.

On the other hand, three are the performance indicators assessed in this article: outage probability, coverage level and maximum, service data rate. For each considered metric, -i.e., IP PER, ESR, and ESR5(20), a user is in outage when experiencing a value greater than $1 \%$. The coverage level for a given configuration represents the percentage of users that are not in outage for this configuration. Finally, the maximum service data rate is defined as the higher data rate that presents a coverage value greater than 95\%. Both coverage level and maximum service data rate are computed for each metric.

\subsection{Performance assessment for different AL-FEC configurations}

In this section, the trade-off between PHY-FEC and ALFEC is investigated along with the influence of the protection period. Figure 5 shows an example of coverage performance of E-MBMS for different AL-FEC configurations, i.e., application code rate and protection period combinations. The left part corresponds to MCS 6 and the right part to MCS 7. In this case, ESR criterion was chosen and only vehicular users were deployed with an ISD scenario of $500 \mathrm{~m}$.

In general, the coverage level depends on the MCS, obtaining better coverage for more robust MCS. Of course, higher robustness comes at the expense of a lower service date rate. According to the figure, without AL-FEC-AL-FEC code rate 1-both MCS 6 and MCS 7 are unable to meet coverage needs. However, the utilization of AL-FEC improves coverage. In particular, the coverage level increases with lower code rates and higher protection periods.

Left part of Figure 6 depicts the coverage level as a function of the protection period for several MCS with fixed AL-FEC code rate. These results reinforce the idea that coverage level increases with more robust MCSs and higher protection periods.

On the other hand, the optimum AL-FEC code rate is different depending on the MCS and protection period values. This is shown on the right part of Figure 6. As it can be seen, the optimum AL-FEC code rate is higher with larger protection periods and more robust MCSs.

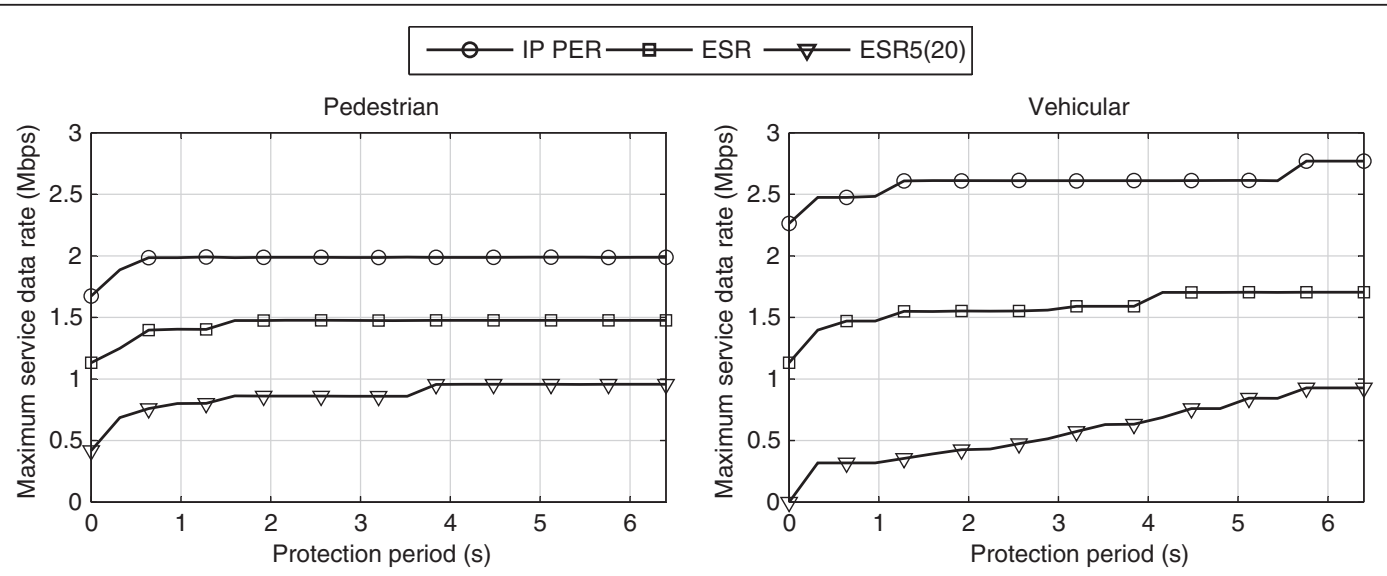

Figure 8 Maximum service data rate versus $T_{\mathrm{pp}}$ for several metrics for pedestrian (left) and vehicular (right) users. 
Table 3 Maximum data rate comparison considering both use and no-use of AL-FEC with $T_{p p}$ of $1.28 \mathrm{~s}$

\begin{tabular}{|c|c|c|c|c|c|c|}
\hline & \multicolumn{3}{|l|}{ Pedestrian } & \multicolumn{3}{|l|}{ Vehicular } \\
\hline & \multicolumn{2}{|c|}{ Maximum data rate (kbps) } & \multirow{2}{*}{$\begin{array}{l}\text { Improvement } \\
\text { (\%) }\end{array}$} & \multicolumn{2}{|c|}{ Maximum data rate (kbps) } & \multirow{2}{*}{$\begin{array}{l}\text { Improvement } \\
\text { (\%) }\end{array}$} \\
\hline & No AL-FEC & AL-FEC & & No AL-FEC & AL-FEC & \\
\hline IP PER & 1672.80 & 1990.63 & 19.00 & 2263.20 & 2609.25 & 15.29 \\
\hline ESR & 1131.60 & 1402.63 & 23.95 & 1131.60 & 1549.63 & 36.94 \\
\hline ESR5(20) & 418.20 & 802.38 & 91.86 & 0 & 355.25 & $\operatorname{lnf}$ \\
\hline
\end{tabular}

These results are tightly related with the maximum service data rate, as discussed below.

Figure 7 shows the maximum service data rate versus the protection period for several MCS using the ESR metric. The results are for both pedestrian and vehicular users. For each MCS, the maximum service data rate is obtained with the maximum Raptor code rate that guarantees a 95\% coverage level. Note that a protection period of $0 \mathrm{~s}$ implies no use of AL-FEC and the maximum Raptor code rate can be different in the same curve depending on the protection period.

As Figure 7 shows, AL-FEC is required if MCSs is greater than 4 . In these cases, the maximum service data rate increases with longer protection periods. For this scenario, the best option is to use the MCS 5 for pedestrian users whereas, for vehicular users, the optimum MCS-among MCS 5, 6, or 7-depends on the protection period. From these results, two conclusions can be drawn. First, for the pedestrian case at some point longer protection periods do not entail any advantage. Second, the benefits of AL-FEC are higher for vehicular users as compared with pedestrian users. The reason for this is that the performance of AL-FEC increases with the speed of the users since it is possible to exploit the temporal diversity within the protection period. This fact also explains the lower maximum service data rate of pedestrian users.

\subsection{AL-FEC performance over different error metrics}

This section studies AL-FEC performance over different error metrics such as IP PER, ESR, and ESR5(20). Given a protection period, the maximum service data rate was obtained choosing the best combination of MCS and AL-FEC code rate.

Figure 8 shows the maximum service data rate for pedestrian (left) and vehicular (right) users. Note that the ESR curves of Figure 8 correspond to the upper envelope of the curves showed in Figure 7. The same conclusions drawn from Figure 7 can be made from Figure 8. However, the more restrictive the quality criterion, the lower the maximum service data rate reached and the higher the improvement achieved using AL-FEC.

Moreover, longer protection periods result in a higher maximum service data rate. However, the introduction of application layer error recovery mechanisms increases the zapping time. This trade-off must be taken into account in the system design, provided that zapping times greater than $2 \mathrm{~s}$ are annoying for the end user. Note that zapping time is equal to 1.5 times the protection period without using fast zapping techniques. If the parity data are transmitted before source data, the zapping time can be reduced. This reduction depends on the AL-FEC code rate. Therefore, in order to show AL-FEC performance for zapping times less than $2 \mathrm{~s}$ several results using a protection period of $1.28 \mathrm{~s}$-which corresponds to a zapping time about $1.92 \mathrm{~s}$-are shown in Table 3. These results are compared with the case in which AL-FEC is not used.

Finally, Table 4 summarizes the best combination-in terms of maximum service data rate-of MCS and ALFEC code rates for different quality criterions and type of users. These results correspond to a protection period of $1.28 \mathrm{~s}$. Two are the main conclusions that can be drawn from these results. First, vehicular users need, in general, less protection than pedestrian users. Second, with a more restrictive quality criterion it is required a higher protection both at physical and application layers. Obviously, the maximum data rate is lower when the quality criterion is more restrictive.

\subsection{Effect of SINR distribution over AL-FEC performance}

This section assesses the performance of AL-FEC when varying the SINR distribution. With this aim two scenarios with different ISDs-500 and $1500 \mathrm{~m}$-were analyzed. For the sake of simplicity, the results are only shown for vehicular users.

The average SINR of users with an ISD of $500 \mathrm{~m}$ is approximately $2 \mathrm{~dB}$ higher than with an ISD of $1500 \mathrm{~m}$. As summarized in Table 5, best SINR conditions allow using lower levels of protection. Provided that the amount

Table 4 The best configurations for pedestrian and vehicular users with $T_{p p}$ of $1.28 \mathrm{~s}$

\begin{tabular}{lllllc}
\hline & \multicolumn{2}{l}{ Pedestrian } & & \multicolumn{2}{l}{ Vehicular } \\
\cline { 2 - 3 } & MCS & AL-FEC CR & & MCS & AL-FEC CR \\
\hline IP PER & 6 & 0.88 & 7 & 0.92 \\
ESR & 5 & 0.83 & 5 & 0.92 \\
ESR5(20) & 4 & 0.70 & 2 & 0.83 \\
\hline
\end{tabular}


Table 5 The best configurations for different ISDs for vehicular users with $T_{\mathrm{pp}}$ of $1.28 \mathrm{~s}$

\begin{tabular}{|c|c|c|c|c|c|c|}
\hline & \multicolumn{3}{|c|}{ ISD $500 \mathrm{~m}$} & \multicolumn{3}{|c|}{ ISD $1500 \mathrm{~m}$} \\
\hline & MCS & AL-FEC CR & Maximum data rate (kbps) & MCS & AL-FEC CR & Maximum data rate (kbps) \\
\hline IP PER & 7 & 0.92 & 2609.25 & 5 & 0.70 & 1182.13 \\
\hline ESR & 5 & 0.92 & 1549.63 & 3 & 0.84 & 594.13 \\
\hline ESR5(20) & 2 & 0.83 & 355.25 & 2 & 0.26 & 110.25 \\
\hline
\end{tabular}

of resources reserved for E-MBMS is the same, this robustness implies that the maximum service data rate is higher for smaller ISD scenarios.

\subsection{Performance assessment for different configurations of the scheduling of E-MBMS}

So far, the results have been obtained assuming that one service channel occupies all the resources allocated to EMBMS and MSP is $320 \mathrm{~ms}$. This section studies the effect of E-MBMS scheduling on AL-FEC performance. The assessment is performed using the example showed in Figure 4, where four streaming channels are multiplexed within the protection period using different values of the MSP. Figure 4 showed that the higher the MSP, the lower the resources dedicated to E-MBMS control. However, a high MSP reduces time diversity. This section analyzes this trade-off.

As explained in Section 5.2, the performance of ALFEC improves with the user velocity, since it is possible to exploit the temporal diversity within the protection period. This is why this section focuses on a vehicular scenario. Besides, two different protection periods-1.28 and $5.12 \mathrm{~s}$-and several MSPs were analyzed. ESR metric was used.

Figure 9 shows the coverage level as a function of the AL-FEC code rate and the MSP. MCS 7 was used for a protection period of $1.28 \mathrm{~s}$ (left) and MCS 8 for a protection period of $5.12 \mathrm{~s}$ (right). On the one hand, using low MSPs implies more resource dedicated to E-MBMS signaling. For example, with a protection period of 1.28 s 16 MSI are transmitted using an MSP of $80 \mathrm{~ms}$, whereas only $4 \mathrm{MSI}$ are required with an MSP of 320 ms. However, the results show that, for a given AL-FEC code rate, the lower the MSP, the higher the coverage level. Indeed, using a low MSP allows for a better exploitation of temporal diversity since each E-MBMS channel transmission is divided into several intervals within the protection period. Despite the additional signaling overhead, this entails a larger effective interleaving and a better performance. In addition, the effect of the MSP is more significant for longer protection periods since there is more room for time interleaving. It is worth noting that this effect depends on the speed of the user, since for low mobility users there is little temporal diversity. In fact, similar simulations were performed for pedestrian users and this effect was not noticeable.

\section{Conclusions}

This article has presented some guidelines for the efficient transmission of E-MBMS services proposing the utilization of AL-FEC protection based on Raptor codes for the transmission of streaming services over LTE networks. With this aim, this article has taken into account the RRM problem and the trade-off between PHY-FEC and AL-FEC. Several AL-FEC configurations with different protection periods and code rates have been compared with the transmission of streaming services without AL-FEC protection. This article has also studied the effect of several AL-FEC parameters on system performance in several scenarios with different average SINRs and the effect on time multiplexing of different services within the AL-FEC time interleaving.
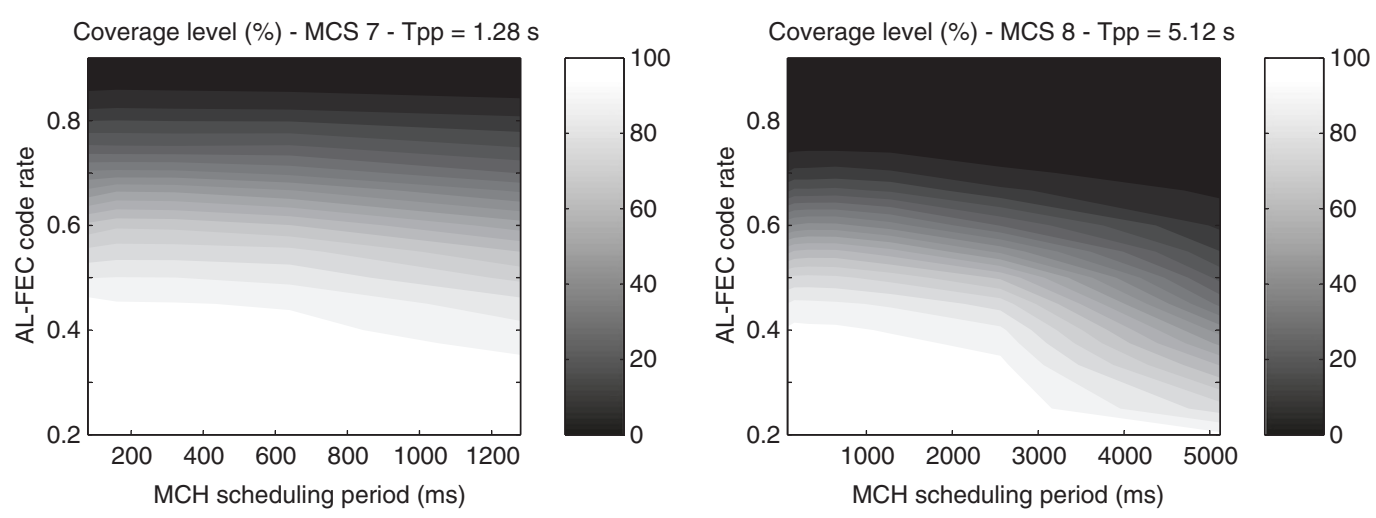

Figure 9 Coverage level versus AL-FEC CR and MSP for MCS 7 with $T_{\mathrm{pp}}$ of $1.28 \mathrm{~s}$ (left) and MCS 8 with $T_{\mathrm{pp}}$ of $5.12 \mathrm{~s}$ (right). 
In the first assessment, several conclusions were obtained. The first one is that the coverage of streaming services increases with the usage of AL-FEC and, then, a higher maximum service data rate can be obtained if AL-FEC is used. However, this gain depends on the parameter settings, the user type scenario, and the quality criterions. The results show that the larger the protection period, the greater the maximum service data rate. However, these large protection periods produce an excessive zapping time that affect to the QoS and it is not recommended for streaming services. For example, using a protection period of $1.28 \mathrm{~s}$ that gives a zapping time lower than $2 \mathrm{~s}$, which can be considered as acceptable, gains of 23.95 and $36.94 \%$ for ESR were obtained using AL-FEC for pedestrian and vehicular users, respectively. It shows that the gain is greater in scenarios with high mobility users. Finally, the more restrictive the quality criterion, the lower the maximum service data rate achieved although the improvement achieved using ALFEC is higher in these criterions (91.86\% using an ESR5 (20) for pedestrian users).

In the others assessments, a higher protection both at physical layer and application layer must be applied in larger ISD scenarios in order to obtain the maximum service data rate. Obviously, the maximum service data rate in this case is lower. Finally, a higher interleaving between different channels within the protection period improves the coverage level as it is possible to exploit better the temporal diversity using low. Also, the effective interleaving is enlarged. However, this effect depends on the speed of the user, since for low mobility users there is little temporal diversity.

\footnotetext{
Abbreviations

3G: Third generation; 3GPP: Third generation partnership project; AL-FEC: Application layer FEC; AWGN: Additive white Gaussian noise; BCCH: Broadcast control channel; BLER: Block error rate; CP: Cyclic Prefix; CR: Code rate; CSA: Common subframe allocation; E-MBMS: Enhanced MBMS; eNodeB: Evolved Node B; EPA: Extended pedestrian A; ESR: Erroneous second ratio; EVA: Extended vehicular A; FEC: Forward error correction; IMT-Advanced: International Mobile TelecommunicationsAdvanced; IP: Internet protocol; ISD: Inter-site distance; ITU-R: ITU Radiocommunication Sector; LTE: Long term evolution; MAC: Medium access control; MBMS: Multimedia broadcast multicast service; MBSFN: MBMS over single frequency networks; $\mathrm{MCCH}$ : Multicast control channel; MCH: Multicast channel; MCS: Modulation and coding scheme; MTCH: Multicast traffic channel; MTU: Maximum transmission unit; MSI: MCH scheduling information; MSP: MCH scheduling period; NAL: Network abstraction layer; OFDMA: Orthogonal frequency division multiple access; PDU: Protocol data unit; PDP: Power delay profile; PER: Packet error rate; PHY: Physical; PMCH: Physical multicast channel; p-tm: Point-to-multipoint; QAM: Quadrature amplitude modulation; QoS: Quality-of-service; QPSK: Quadrature phase shift keying; RLC: Radio link control; RRM: Radio resource management; RTP: Real-time transport protocol; SFN: Single frequency network; SIB: System information block; SINR: Signal-to-interference plus noise ratio; TTI: Transmission time interval; UMTS: Universal mobile telecommunications system; WINNER+: Wireless world initiative new radio +.
}

\section{Competing interests}

The authors declare that they have no competing interests.

\section{Acknowledgments}

This study was supported by the Spanish Ministry of Science under the project TEC2011-27723-C02-02.

Received: 26 September 2012 Accepted: 12 February 2013

Published: 15 March 2013

\section{References}

1. 3GPP TS 25.346 V6.4.0, Introduction of the Multimedia Broadcast Multicast Service (MBMS) in the Radio Access Network (RAN); Stage 2, 2005.

2. H Deng, $X$ Tao, J Lu, Qos-aware resource allocation for mixed multicast and unicast traffic in OFDMA networks. EURASIP Journal on Wireless Communications and Networking 2012(195), 1-10 (2012). doi:10.1186/16871499-2012-195

3. 3GPP TS 26.346 V9.5.0, Multimedia Broadcast/Multicast Service (MBMS); Protocols and codecs, 2011.

4. A Shokrollahi, Raptor codes. IEEE Transactions on Information Theory 52(6), 2251-2567 (2006). doi:10.1109/TIT.2006.874390

5. 3GPP TS 25.346 V7.5.0, Introduction of the Multimedia Broadcast/Multicast Service (MBMS) in the Radio Access Network (RAN); Stage 2, 2007.

6. D Martín-Sacristán, JF Monserrat, J Cabrejas, D Calabuig, S Garrigas, N Cardona, On the way towards fourth-generation mobile: 3GPP LTE and LTEadvanced. EURASIP Journal on Wireless Communications and Networking, 1-10 (2009). doi:10.1155/2009/354089

7. 3GPP TS 36.211 V.8.5.0, Evolved Universal Terrestrial Radio Access (E-UTRA); Physical Channels and Modulation, 2008.

8. 3GPP TS 36.300 V9.1.0, Evolved Universal Terrestrial Radio Access (E-UTRA) and Evolved Universal Terrestrial Radio Access Network (E-UTRAN); Overall description, 2009.

9. JF Monserrat, J Calabuig, A Fernandez-Aguilella, D Gomez-Barquero, Joint delivery of unicast and E-MBMS services in LTE networks. IEEE Transactions on Broadcasting. 58(2), 157-167 (2012). doi:10.1109/TBC.2012.2191030

10. A Alexiou, C Bouras, V Kokkinos, A Papazois, G Tsichritzis, in Wireless MultiAccess Environments and Quality of Service Provisioning: Solutions and Application, Multimedia broadcasting in LTE networks, ed. by GM Muntean, R Trestian (IGI Global, Hershey, PA, 2012), pp. 269-289

11. N Wang, Z Zhang, The impact of application layer Raptor FEC on the coverage of MBMS. Radio and Wireless Symposium, 2008 IEEE, 223-226 (2008). doi:10.1109/RWS.2008.4463469

12. D Gomez-Barquero, A Fernandez-Aguilella, N Cardona, Multicast delivery of file download services in evolved $3 G$ mobile networks with HSDPA and MBMS. IEEE Transactions on Broadcasting. 55(4), 742-751 (2009). doi:10.1109/TBC.2009.2032800

13. T Stockhammer, A Shokrollahi, M Watson, M Luby, T Gasiba, in Handbook of Mobile Broadcasting: DVB-H, DMB, ISDB-T and Media FLO, Application layer forward error correction for mobile multimedia broadcasting, ed. by B Furhet, S Ahson (CRC Press, Boca Raton, FL, 2008), pp. 239--280

14. J Afzal, T Stockhammer, T Gasiba, W Xu, Video streaming over MBMS: a system design approach. Journal of Multimedia. 1(5), 25-35 (2006)

15. A Alexiou, C Bouras, V Kokkinos, A Papazois, G Tseliou, in Cellular Networks Positioning, Performance Analysis, Reliability, Forward error correction for reliable e-MBMS transmissions in LTE networks, ed. by A Melikov (InTech, Rijeka, Croatia, 2011), pp. 353-374

16. D Munaretto, D Jurca, J Widmer, Broadcast video streaming in cellular networks: An adaptation framework for channel, video and AL-FEC rates allocation. Wireless Internet Conference (WICON), 2010 The 5th Annual ICST, 1-9 (2010)

17. C Bouras, N Kanakis, V Kokkinos, A Papazois, Application layer forward error correction for multicast streaming over LTE networks. Int. J. Commun. Syst (2012). doi:10.1002/dac.2321

18. RaptorQ technical overview, Qualcomm Technical Report (2010). http:// www.qualcomm.com/instella_api/asset/3cd5b620-afea-012d-72bc$12313804 \mathrm{dc} 61$

19. T Mladenov, K Kim, S Nooshabadi, Forward error correction with RaptorQ Code on embedded systems. Circuits and Systems (MWSCAS), 2011 IEEE 54th International Midwest Symposium, 1-4 (2011). doi:10.1109/ MWSCAS.2011.6026424

20. J Calabuig, JF Monserrat, D Martín-Sacristán, J Olmos, Comparison of multicast/broadcast services in Long Term Evolution Advanced and IEEE 802.16m networks. Wirel. Commun. Mob. Comput. (2012). doi:10.1002/ wcm. 2229 
21. X Jiang, G Zhu, W Wu, Y Gao, Design of LTE E-MBMS Dynamic Scheduling Information. Wireless Communications Networking and Mobile Computing (WiCOM), 2010 6th International Conference on, 1-5 (2010). doi:10.1109/ WICOM.2010.5600210

22. 3GPP TS 36.331 V.9.9.0, Evolved Universal Terrestrial Radio Access (E-UTRA); Radio Resource Control (RRC); Protocol Specification, 2011.

23. M-L Alberi Morel, S Kerboeuf, B Sayadi, Y Leprovost, F Faucheux, Performance Evaluation of Channel Change for DVB-SH Streaming Services. Communications (ICC), 2010 IEEE International Conference on, 1-6 (2010). doi:10.1109/ICC.2010.5502523

24. WINNER + MT-Advanced Calibration: Guidelines, software and results, 2009. http://projects.celtic-initiative.org/winner+/WINNER+\%20Evaluation\% 20Group.html

25. K Brueninghaus, D Astely, T Salzer, S Visuri, A Alexiou, S Karger, GA Seraji, Link performance models for system level simulations of broadband radio access systems, in Proceedings of 16th IEEE International Symposium on Personal, Indoor and Mobile Radio Communications (PIMRC). Berlin, Germany 4, 2306-2311 (2005). doi:10.1109/PIMRC.2005.1651855

26. ITU-R M.2135, Guidelines for evaluation of radio interface technologies for IMT Advanced, 2008. http://www.itu.int/dms_pub/itu-r/opb/rep/R-REP-M.21352008-PDF-E.pdf

27. 3GPP TS 36.101 V.9.10.0, Evolved Universal Terrestrial Radio Access (E-UTRA); User Equipment (UE) radio transmission and reception, 2011

28. L Rong, O Ben Haddada, S-E Elayoubi, Analytical Analysis of the Coverage of a MBSFN OFDMA Network," Global Telecommunications Conference. IEEE GLOBECOM 2008. IEEE, 1-5 (2008). doi:10.1109/GLOCOM.2008.ECP.459

29. 3GPP TSG-SA WG4 S4-100861, Relation between MBSFN area and intended MBMS service reception area, 2010.

30. 3GPP TR 36.213 V.9.3.0, Evolved Universal Terrestrial Radio Access (E-UTRA); Physical layer procedures, 2010.

doi:10.1186/1687-1499-2013-73

Cite this article as: Calabuig et al:: AL-FEC for streaming services in LTE E-MBMS. EURASIP Journal on Wireless Communications and Networking 2013 2013:73.

\section{Submit your manuscript to a SpringerOpen ${ }^{\circ}$ journal and benefit from:}

- Convenient online submission

- Rigorous peer review

- Immediate publication on acceptance

- Open access: articles freely available online

- High visibility within the field

- Retaining the copyright to your article

Submit your next manuscript at $\gg$ springeropen.com 